\title{
Predictive Models and Algorithms for the Need of Transfusion Including Massive Transfusion in Severely Injured Patients
}

\author{
Marc Maegele ${ }^{a, b} \quad$ Thomas Brockamp $^{b} \quad$ Ulrike Nienaber ${ }^{c} \quad$ Christian Probst $^{a}$ \\ Herbert Schoechl $^{d} \quad$ Klaus Görlinger $^{\mathrm{e}}$ Philip Spinella $^{f}$

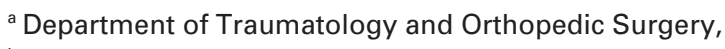 \\ ${ }^{\mathrm{b}}$ Institute for Research in Operative Medicine (IFOM), Cologne-Merheim Medical Center (CMMC), University of Witten/Herdecke, Cologne, \\ ${ }^{c}$ Akademie der Unfallchirurgie GmbH, Berlin, Germany \\ ${ }^{\mathrm{d}}$ Department of Anesthesiology and Intensive Care, AUVA Trauma Hospital, Salzburg, Austria \\ ${ }^{\text {e }}$ Department of Anesthesiology and Intensive Care, University of Essen Medical Center, Essen, Germany \\ ${ }^{f}$ St. Louis Children's Hospital, Director Critical Care Translational Research Program, Washington University School of Medicine, \\ St. Louis, MO, USA
}

\section{Keywords}

Trauma $\cdot$ Hemorrhage $\cdot$ Prediction $\cdot$ Models $\cdot$ Transfusion

\section{Summary}

Background: Despite improvements on how to resuscitate exsanguinating patients, one remaining key to improve outcome is to expeditiously and reproducibly identify patients most likely to require transfusion including massive transfusion (MT). This work summarizes yet developed algorithms/scoring systems for transfusion including MT in civilian and military trauma populations. Methods: A systematic search of evidence was conducted utilizing OVID/MEDLINE (1966 to present) and the 'Medical Algorithms Project'. Results and Conclusions: The models developed suggest combinations of physiologic, hemodynamic, laboratory, injury severity and demographic triggers identified on the initial evaluation of the bleeding trauma patient. Many approaches use a combination of dichotomous variables readily accessible after arrival but others rely on time-consuming calculations or complex algorithms and may have limited real-time application. Weighted and more sophisticated systems including higher numbers of variables perform superior. A common limitation to all models is their retrospective nature, and prospective validations are urgently needed. Point-of-care viscoelastic testing may be an alternative to these systems.

\section{Schlüsselwörter}

Trauma · Blutung · Prädiktion · Modelle · Transfusion

\section{Zusammenfassung}

Hintergrund: Trotz deutlicher Verbesserungen im klinischen Management schwerstverletzter und blutender $\mathrm{Pa}$ tienten ist die Früherkennung und die Identifikation von Risikopatienten für Bluttransfusionen einschließlich der Massentransfusion nach wie vor eine Herausforderung in der Akutphase. Die vorliegende Arbeit vermittelt eine Übersicht über bislang erarbeitete Modelle und Algorithmen zur Früherkennung und Stratifizierung von Risikopatienten sowohl aus dem militärischen als auch aus dem zivilen Traumabereich. Methoden: Eine systematische Suche unter Einschluss von OVID/MEDLINE (1966 bis heute) und dem "Medical Algorithms Project» wurde durchgeführt. Ergebnisse und Schlussfolgerungen: Die hierbei identifizierten Modelle beinhalten in der Regel Kombinationen frühzeitig nach Schockraumaufnahme verfügbarer physiologischer, hämodynamischer und laborchemischer Daten sowie Informationen zu Verletzungsschwere und Demographie. Viele Ansätze benutzen Kombinationen rasch verfügbarer dichotomer Variablen, andere hingegen zeitaufwendige Kalkulationen mit sicherlich limitierter Anwendbarkeit in der klinischen Akutphase. Gewichtete und komplexere Systeme unter Einschluss mehrerer Variablen schneiden im Vergleich besser ab. Eine allgegenwärtige Limitierung sämtlicher Ansätze ist die retrospektive Natur ihrer Entwicklung und Validierung, und die prospektive Validierung ist dringend notwendig. Viskoelastische Testverfahren bieten möglicherweise eine Alternative.

\section{KARGER \\ Fax +497614520714 \\ Information@Karger.de}

www.karger.com
(C) 2012 S. Karger GmbH, Freiburg $1660-3796 / 12 / 0392-0085 \$ 38.00 / 0$

Accessible online at: www.karger.com/tml
Prof. Dr. med. Marc Maegele

Department of Traumatology and Orthopedic Surgery

Cologne-Merheim Medical Center (CMMC), University of Witten/Herdecke

Ostmerheimerstr. 200, 51109 Köln, Germany

Tel. +49 221-8907 13614; Fax -989 5721

Marc.Maegele@t-online.de 


\section{Introduction}

Trauma is the leading cause of death worldwide in persons under the age of 40 years [1] and accounts for approximately $10 \%$ of all deaths in general [2]. Despite substantial improvements in acute trauma care, uncontrolled hemorrhage is still responsible for more than $50 \%$ of all trauma-related deaths in both civilian and military settings within the first $48 \mathrm{~h}$ after hospital admission [3]. Clinical observations together with recent research highlighted the central role of coagulopathy in acute trauma care [4-10], but rapid identification of patients with active ongoing bleeding requiring transfusion or even massive transfusion (MT) remains unsatisfactory. Substantial problems include delayed turn-around times for conventional coagulation testing, incomplete characterization, and their poor predictive nature not accurately reflecting the patient's true coagulation status $[9,11]$. Although international normalized ratio (INR) and base deficit (BD) are good predictors of mortality, by themselves, they cannot discriminate between patients to may go or not go on for MT. Second, surgical relevant bleeding due to thoracic and/or retro-/ intraperitoneal organ injury is difficult to detect and often requires time-consuming diagnostics [12]. Thus, significant hemorrhage and coagulopathy may be underestimated or even missed during early resuscitation $[13,14]$.

Death from traumatic exsanguination usually occurs early, typically within the first 6-12 h after initial impact [15-18]. Approximately $10 \%$ of all trauma patients are transfused with at least one unit of blood, and up to $30 \%$ of these require MT as defined as transfusion of $\geq 10$ units within the first $24 \mathrm{~h}$ after emergency room admission $[19,20]$. On average, one out four trauma patients to arrive in the trauma bay is already coagulopathic upon admission [8-10,13] but incidence rates up to $60 \%$ have been reported according to definition [21]. Thus, the early identification of trauma patients at risk for transfusion and MT is of fundamental clinical importance in order to rapidly address and correct the acute coagulopathy of trauma, including potential triggers, for example acidosis, and hypothermia, to allow early activation of MT protocols and to allow early mobilization of resources, for example blood bank resources in the civilian setting as well as activation of whole blood donation in the military setting $[22,23]$. In this issue of Transfusion Medicine and Hemotherapy, Meißner and Schlenke [58] provide an excellent review of the current literature on fluid resuscitation, administration of blood products, and hemostatic agents in massive hemorrhage in order to optimize patients' blood and coagulation management during acute care.

Meanwhile, several authors have shown that early recognition of the acute coagulopathy of trauma accompanied by adequate and aggressive management including the balanced use of blood components can correct coagulopathy, control bleeding, reduce blood product use, and improve outcome in severely injured patients $[24,25]$. Predictive models for MT

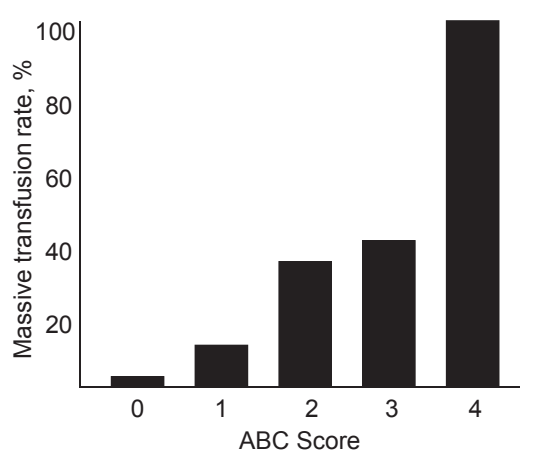

\begin{tabular}{|ll|}
\hline Penetrating mechanism & 1 \\
ER systolic blood pressure $\leq 90 \mathrm{~mm} \mathrm{Hg}$ & 1 \\
ER heart rate $\geq 120 \mathrm{bpm}$ & 1 \\
FAST positiv & 1 \\
\hline
\end{tabular}

Rate of massive transfusion by ABC Score.

have been successfully developed and implemented for cardiac and liver transplant surgery [26, 27]. The included variables, however, reflect the contrast between the controlled environment of a planned intervention versus the rather unexpected scenario in acute trauma surgery.

\section{Methods}

The present work summarizes and discusses the yet developed and published algorithms and scoring systems for transfusion including MT in both civilian and trauma populations. To develop this review, a systematic search of available scientific evidence was conducted utilizing OVID/MEDLINE (1966 to present) and the 'Medical Algorithms Project' ( $w w w$. medal.org) with selected search terms including combinations of flowing key words and terms. In addition, reference lists and bibliographies were analyzed for additional relevant manuscripts.

\section{Scoring Systems}

\section{Models Based upon Data from Civilian Trauma Patients}

\section{Assessment of Blood Consumption (ABC) Score [28]}

This model was developed based upon a retrospective cohort $(\mathrm{n}=596)$ of primary civilian trauma patients (level I major trauma activations) admitted to a single level I trauma center in the USA (2005-2006) and who had survived at least $30 \mathrm{~min}$ after arrival and who had received any blood transfusions during their hospitalization [28]. The score, which uses non-laboratory and non-weighted parameters (fig. 1, table 1), was created by the institution's trauma faculty based upon their clinical experience of appropriate activation of the trauma center's protocol. Multiple logistic regression modeling evaluated four 


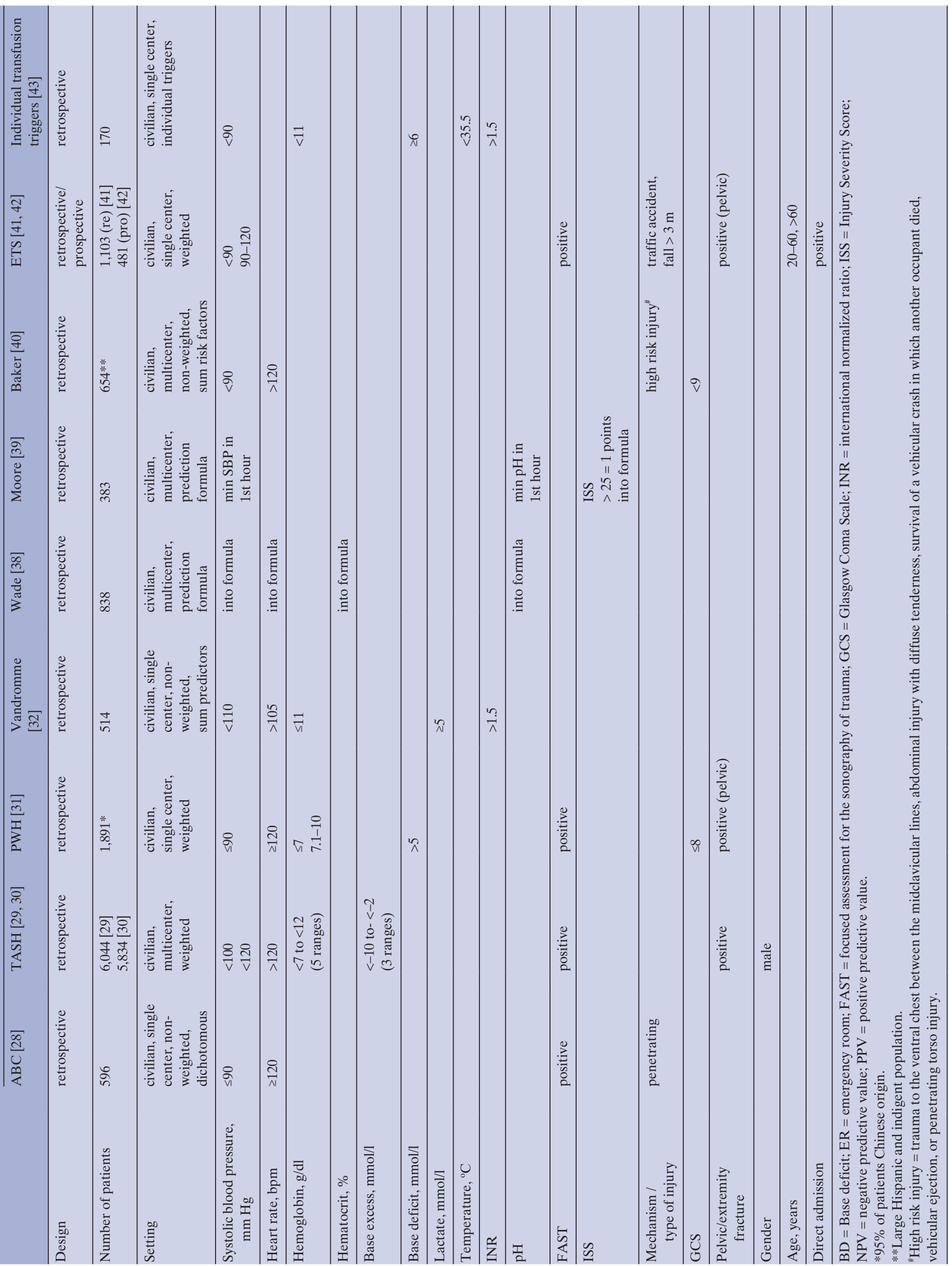




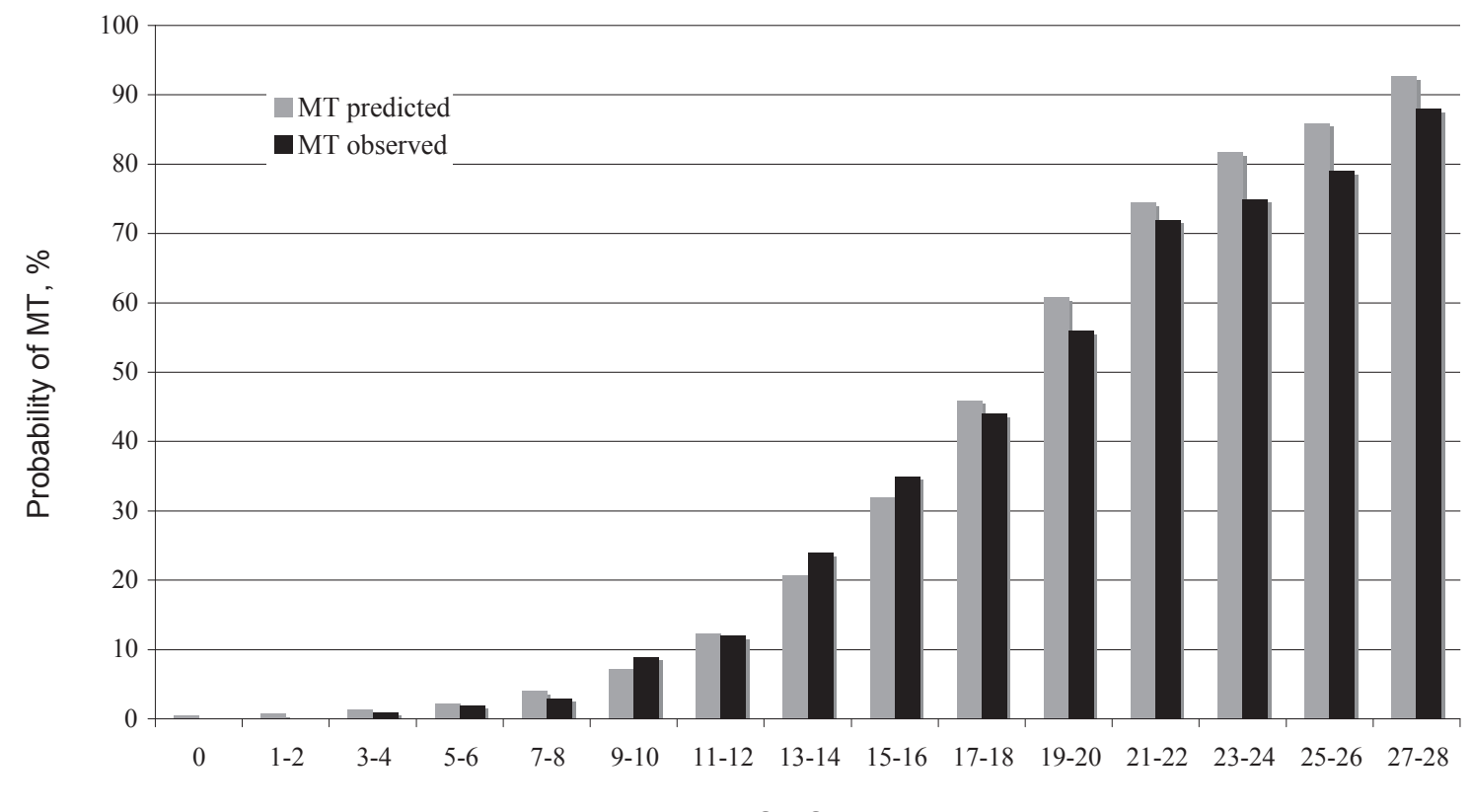

TASH Score

\begin{tabular}{lll}
\hline Hemoglobin, g/dl & $<7$ & 8 \\
& $<9$ & 6 \\
& $<10$ & 4 \\
& $<11$ & 3 \\
& $<12$ & 2 \\
Base excess, mmol/l & $<-10$ & 4 \\
& $<-6$ & 3 \\
Systolic blood pressure, mm Hg & $<-2$ & 1 \\
Clinically instable pelvic fracture & $<120$ & 1 \\
Open/dislocated femur fracture & 3 & \\
Heart rate, bpm & & \\
FAST + & & \\
\hline
\end{tabular}

Fig. 2. The Trauma-Associated Severe Hemorrhage (TASH) Score: Predicted and observed rates for massive transfusion (MT) with increasing TASH Scores. overall accuracy of the $\mathrm{ABC}$ score by area under the receiver operator curve (AUROC) was 0.859. An ABC score of 2 or greater is supposed to be $75 \%$ sensitive and $86 \%$ specific for predicting MT.

\section{Trauma-Associated Severe Hemorrhage (TASH) Score} [29, 30]

The TASH Score was initially developed and validated on the basis of data from 6,044 severely injured blunt trauma patients derived from a multicenter civilian trauma database run 


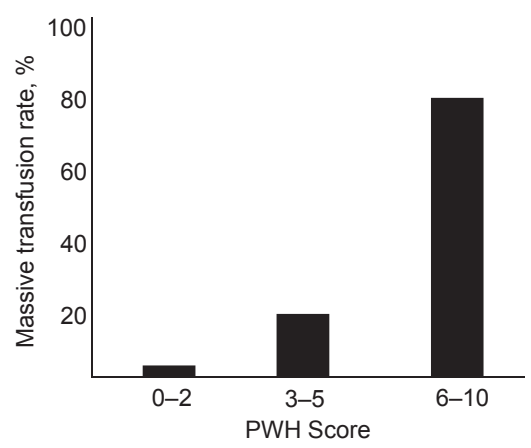

\begin{tabular}{|lc|}
\hline ER heart rate $>120 \mathrm{bpm}$ & 1 \\
ER systolic blood pressure $\leq 90 \mathrm{~mm} \mathrm{Hg}$ & 1 \\
Glasgow Coma Scale $\leq 8$ & 1 \\
Displaced pelvic fracture & 1 \\
CT scan or FAST + for fluid & 1 \\
Base deficit $<5 \mathrm{mmol} / \mathrm{l}$ & 1 \\
Hemoglobin $\leq 7 \mathrm{~g} / \mathrm{dl}$ & 10 \\
Hemoglobin $7.1-10 \mathrm{~g} / \mathrm{dl}$ & 1 \\
\hline
\end{tabular}

nationwide by the German Society for Trauma Surgery (Trauma Registry of the Deutsche Gesellschaft für Unfallchirurgie (TR-DGU)) [29]. Recently, the performance of the TASH Score was internally re-validated on data from 5,834 patients derived from the 2004-2007 TR-DGU database [30]. For this update, potential clinical and laboratory variables documented in the registry were again subjected to bivariate and multivariate logistic regression analysis to predict the probability for MT during early in-hospital resuscitation as a surrogate for ongoing life-threatening hemorrhage. MT was defined as the administration of at least 10 units of pRBCs between emergency room and intensive care unit (ICU) admission. In summary, TASH uses seven independent but weighted variables to identify patients who will require a MT: systolic blood pressure, gender, hemoglobin, FAST exam, heart rate, base excess $(\mathrm{BE})$, and extremity or pelvic fractures (fig. 2, table 1). The possible range of the scores is between 0 and 28 where each point corresponds to a risk for MT in percent. The TASH Score is transformed into a probability for MT using a the following logistic function:

$$
\mathrm{p}=1 /[1+\exp (5.4-0.3 \times \mathrm{TASH})]
$$

By its recent update, the high performance of the score was not only restored but enhanced, as reflected by an increased AUROC of 0.905 . At a cut-off of $>16$ out of 28 points the correct classification rate is $>90 \%$.

Prince of Wales Hospital / Rainer Score (PWH Score) [31] This model was developed on the basis of a retrospective analysis of 1,891 civilian trauma patients (2001-2009) derived from a single center administrative trauma database (PWH Trauma Registry) [31]. The Prince of Wales Hospital (PWH) is a university tertiary referral center located in the New Territories of Hong Kong where $95 \%$ of the population are Chinese. 92 patients required $\geq 10$ units of pRBCs within $24 \mathrm{~h}$ and met the criteria for MT. Univariate analysis followed by multivariate stepwise logistic regression identified seven variables to predict the need for MT: heart rate $\geq 120 \mathrm{bpm}$, systolic blood pressure $\leq 90 \mathrm{~mm} \mathrm{Hg}$, Glasgow Coma Scale (GCS) $\leq 8$, displaced pelvic fracture, CT scan or FAST positive for fluid, $\mathrm{BD}<5 \mathrm{mmol} / \mathrm{l}$, hemoglobin $\leq 7 \mathrm{~g} / \mathrm{dl}$, and hemoglobin 7.1-10 g/dl (fig. 3, table 1). Variables were selected based partly on previous published work and expert opinion regarding their relevance to transfusion and ease of management in the emergency room department. Weighted scores were assigned to each variable according to their adjusted odds ratio. At a cutoff of $\geq 6$ points, the overall correct classification for predicting the need for MT is $96.9 \%$ while the incidence of MT with this value is $82.9 \%$. The AUROC for predicting the need for MT using this model is 0.889 .

\section{Vandromme Score [32]}

The score suggested by Vandromme and colleagues [32] stems from a retrospective analysis of datasets from civilian trauma patients admitted to a single verified level I trauma center in the USA. Based upon three previous studies of MT in the combat setting, clinical characteristics associated with the need for MT were extracted from medical records [33-35]. Given the experience of the authors with blood lactate (BL) in predicting the need for significant transfusion, this parameter was added to the list [36]. The authors have used a classical split data approach with 306 patients extracted from their database to build the development dataset (2005-2007) and 208 patients to build the validation cohort (2007-2008; total $n$ $=514$ patients). MT was defined by the administration of at least 10 units of pRBCs within $24 \mathrm{~h}$ of admission. Univariate as well as multiple round multivariate analyses were conducted for selection of the best-fit predictive model for the association between admission clinical characteristics and the need for $\geq$ pRBCs within $24 \mathrm{~h}$ of admission; the HosmerLemeshow (HL) goodness-of-fit test was used to determine the best-fit model with lower values of the HL statistic suggesting better model fit. Clinical measurements used to create the model included: $\mathrm{BL} \geq 5 \mathrm{mmol} / \mathrm{l}$, heart rate $>105 \mathrm{bpm}$, INR $>1.5$, hemoglobin $\leq 11 \mathrm{~g} / \mathrm{dl}$, and systolic blood pressure $<110$ $\mathrm{mm} \mathrm{Hg}$ (table 1). The best-fit predictive model included $\geq 3$ positive clinical measures (sensitivity: 53\%, specificity: $98 \%$, PPV (positive predictive value): 33\%, NPV (negative predictive value): 99\%). There was increasing PPV with increasing numbers of positive measurements, and the multivariate model including at least four of the five positive clinical predictors resulted in an overall high AUC (0.91 or greater). The discrepancy between the relatively high specificity and NPV in contrast to the relatively low sensitivity and PPV, or the 
usefulness in correctly classifying patients who will need a MT has been attributed to the low MT rate observed in this study ( $2.4 \%)$, because PPV is greatly affected by the prevalence of the disease in a given population [37]. All combinations or clinical measures alone yielded lower predictive probability.

\section{Wade Model [38]}

The Wade Model was developed retrospectively on data obtained from 17 level I trauma centers across the USA collected for 1,574 patients that had received a transfusion during a 1-year period [38]. Multivariate logistic regression was used to predict the need for MT from data routinely available upon emergency room admission. The model to predict whether or not a MT is required was:

$$
\begin{aligned}
& \log [\pi /(1-\pi)]=\beta_{1} \times \mathrm{SBP}+\beta_{2} \times \mathrm{HR}+\beta_{3} \times \mathrm{pH}+ \\
& \beta_{4} \times \mathrm{HCT}+\beta_{0}
\end{aligned}
$$

where the covariates were systolic blood pressure (SBP), heart rate (HR), $\mathrm{pH}$, and hematocrit (HCT) and $\pi$ was the probability of MT given the values of the covariates (table 1 ). Fitting the model on the retrospective database gave $\beta_{1}=$ $-0.0113, \beta_{2}=0.0158, \beta_{3}=0.0932, \beta_{4}=-0.0395$, and $\beta_{0}=32.8738$, based on patients with non-missing values for the covariates $(\mathrm{n}=838)$. According to this model, a patient was (not) predicted to require MT if $\pi>0.5(\leq 0.5)$, resulting in $240(29 \%)$ patients positively predicted (PP) to require MT and 598 (71\%) negatively predicted (NP) to require MT. Of the 838 patients, $322(38 \%)$ actually received MT. This model yielded a PP value of $75 \%$ and an NP value of $72 \%$, with a sensitivity of $87 \%$ and a specificity of $53 \%$.

\section{Moore Model [39]}

This model was developed retrospectively using data from a unique prospective multicenter ( 7 level I trauma centers in the USA) database including major trauma patients without severe brain injury who arrived to the trauma bay in the state of shock [39]. There were 383 database patients, 2 out of 3 had sustained a blunt trauma, of which 93 (24\%) had received a MT (defined as pRBC transfusion volume $>3,000 \mathrm{ml}$ during the first $6 \mathrm{~h}$ after admission). Stepwise multivariate logistic regression analysis revealed the final best fit model which includes: Injury Severity Score (ISS) $\leq 25$ gives 0 points; ISS > 25 gives 1 point, minimum $\mathrm{pH}$ during the first hour of arrival, and minimum systolic blood pressure during the first hour after arrival in $\mathrm{mm} \mathrm{Hg}$ (table 1). The probability (p) of MT is calculated via the following predictive equation:

$$
\begin{aligned}
& \log [\mathrm{p} /(1-\mathrm{p})]=42.1+[0.7 \times(\text { points for ISS })] \\
& -[5.6 \times(\text { min } \mathrm{pH} \text { during } 1 \mathrm{st} \text { hour })]-[0.04 \times \\
& (\text { min SBP during } 1 \text { st hour })]
\end{aligned}
$$

The performance of the score was expressed via the AUROC of 0.804 .
Fig. 4. The Baker Model: Patients with all four risk factors present upon arrival had a $100 \%$ transfusion rate, three out of four risk factors $68 \%$, two out of four $42 \%$, one out of four $12 \%$, and patients with $\mathrm{ab}$ sence of any of the four risk factors had a $2 \%$ transfusion rate. A high risk injury was defined as trauma to the ventral chest between the midclavicular lines, abdominal injury with diffuse tenderness, survival of a vehicular crash in

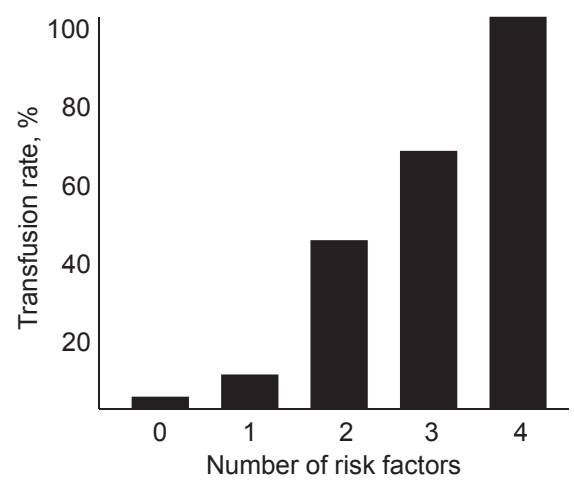

which another occu-

pant died, vehicular ejection, or penetrating torso injury.

\section{Baker Model [40]}

The Baker Model uses a combination of physiologic scoring data and injury severity that are readily accessible and easily obtained upon arrival in the trauma bay to assess the need for transfusion [40]. The model is based upon a retrospective review of 654 consecutive trauma patients over a 6-month period (1996-1997) using emergency department records and data for type and cross-match ( $\mathrm{T}$ and $\mathrm{C}$ ) and emergent blood transfusion which was defined as transfusion within $24 \mathrm{~h}$ of presentation at the emergency department. The setting was a single large public hospital and level I trauma center in the USA serving a large Hispanic and indigent population. Multivariate analysis was performed to identify significant risk factors for emergent blood transfusion, and a model was developed for predicting the probability of transfusion on the basis of log odds ratios of predictive values. Baker and colleagues [40] identified four risk factors for transfusion after injury: systolic blood pressure $<90 \mathrm{~mm} \mathrm{Hg}$, heart rate $>120 \mathrm{bpm}$, GCS $<9$, and high-risk injury (trauma to the ventral chest between the midclavicular lines, abdominal injury with diffuse tenderness, survival of a vehicular crash in which another occupant died, vehicular ejection, or penetrating torso injury (fig. 4, table 1)). Patients with all four risk factors present upon arrival had a $100 \%$ transfusion rate, three out of four risk factors $68 \%$, two out of four $42 \%$, one out of four $12 \%$, and patients with absence of any of the four risk factors had a $2 \%$ transfusion rate with no emergent transfusions occurring in the emergency department. In this series, systolic blood pressure $<90 \mathrm{~mm} \mathrm{Hg}$ demonstrated the highest relative risk for transfusion.

\section{Emergency Transfusion Score (ETS) [41, 42]}

The ETS was developed retrospectively via multivariate logistic regression on data from 1,103 emergency room trauma pa- 
tients [41] and prospectively validated on data from another subset of 481 emergency room patients [42] admitted to a single level I trauma center in Germany over 4 years. A set of nine parameters with possible predictive value for the need of blood transfusion was recorded. All relevant data to calculate this score can be acquired during the first $10 \mathrm{~min}$ after arrival in the trauma bay. The data underwent logistic regression for correlation and the calculation of predictive power. To transform the model into a practical score, all coefficients were rounded. The predictive power of the score was evaluated based on linear regression equation. This score totals up to 9.5 points including the following variables: systolic blood pressure $<90 \mathrm{~mm} \mathrm{Hg}$ (2.5 points), systolic blood pressure 90-120 $\mathrm{mm} \mathrm{Hg}$ (1.5 points), FAST positive (2.0 points), clinically unstable pelvic ring fracture (1.5 points), age 20-60 years ( 0.5 points), age $>60$ years (1.5 points), admission from scene (1.0 points), traffic accident (1.0 points), fall from $>3$ meters (1.0 points (table 1)). The probability for transfusion increased exponentially with the sum of ETS points, e.g. from $0.7 \%$ at one point to $5 \%$ at three points to $97 \%$ at the maximum of 9.5 points. To establish a practical cut-off point (probability / risk of transfusion $\leq 5 \%$ ) a low-risk group was defined at ETS $\leq 3$ (64\% of the entire study group). The sensitivity of the score was calculated at $97.5 \%$ with a specificity of $68 \%$. The PPV was 0.222 , the NPV was 0.998 .

\section{Predictive Value of Individual Transfusion Triggers [43]}

In their recent work, Callcut and colleagues [43] challenged the use of military MT triggers for the civilian population and hypothesized that these triggers may not have equal predictability for MT and that a better understanding of the contribution of each would improve the ability to initiate MT protocols earlier. In the clinical setting, patients with significant hemorrhage are likely to display a variable number of triggers, and more precisely defining the predictive utility of each individual criterion to determine whether victims will require MT or not would substantially support the clinical management of acute trauma hemorrhage including appropriate activation of MT protocols. For this purpose, datasets from 170 consecutive trauma patients admitted to an urban university-based level I trauma center in the mid-western USA over a 1-year period (October 2007 to September 2008) requiring immediate major operative intervention were retrieved from an institutional trauma and transfusion database and retrospectively reviewed using multivariate logistic regression techniques. Transfusion triggers assessed included: systolic blood pressure $<90 \mathrm{~mm}$ $\mathrm{Hg}$, hemoglobin $<11 \mathrm{~g} / \mathrm{dl}$, temperature $<35.5^{\circ} \mathrm{C}$, INR $>1.5$, and $\mathrm{BD} \geq 6 \mathrm{mmol} / \mathrm{l}$ (table 1 ). The cohort was examined to determine the amount of blood transfused solely on these individual proposed transfusion triggers. Although a civilian cohort of patients was assessed, the principle mechanism of injury in these patients was penetrating $(69 \%)$. Transfusion of pRBCs was observed in $45 \%$ (77/170), with the mean number of transfused units being highest in those patients meeting systolic blood pressure (12.9 units) or INR (12.3 units) triggers. Importantly, in comparing between those who met and those who did not met each trigger, there was a statistically significant difference in total $\mathrm{pRBCs}$ received or a trend towards significance in each criteria (except temperature) even when the total volumes of pRBCs fell below the MT thresholds. Overall, the individual triggers did not equally contribute to the predictive value for the need of transfusion. The individual triggers each had a high specificity (except BD) and NPV for the need for early MT with INR exceeding a $95 \%$ specificity and $92 \%$ NPV. The BD was the least frequently ( $44 \%$ of the cohort) obtained parameter of the five triggers and, thus, specificity was relatively poor. The accuracy for predicting the need for MT for the individual triggers was generally high with INR, predicting correctly in $88 \%$ of the time. The individual triggers had different utilities for determining who received MT, with INR (odds ratio 11.3 (95\% CI 2.7-47)) and systolic blood pressure (odds ratio 8.5 (95\% CI 3.4-21)) being highly predictive. The likelihood of receiving any transfusion was also greatest when the INR trigger was exceeded (odds ratio 16.7 (95\% CI2-137)) while the least predictive criterion was temperature (odds ratio 3.4 (95\% CI 1.5-7.7)). To account for the colinear nature of INR, the likelihood of transfusion was also determined for INR and each other variable. The highest likelihood of needing a transfusion was observed in those patients matching both INR and systolic blood pressure criteria (odds ratio 10.4 (95\% CI 2.937.6)), followed by INR and hemoglobin (odds ratio $5.2(95 \%$ CI 2.1-13.1)), and INR and temperature (odds ratio $4.6(95 \%$ CI 2.0-10.6)). Using an equal weighting approach in 53 patients $(31 \%)$ in whom all five triggers were known, when any three or more triggers were exceeded, there was a marked increase in pRBCs transfused.

\section{Models Based upon Data from Military Trauma Patients}

\section{McLaughlin Score [34]}

The model was developed in the military setting based upon a retrospective cohort $(\mathrm{n}=302)$ from a single combat support hospital using univariate and multivariate analyses. Four independent risk factors for MT were identified: heart rate $>105$ bpm, systolic blood pressure $<110 \mathrm{~mm} \mathrm{Hg}, \mathrm{pH}<7.25$, and hematocrit $<32 \%$ [34] (fig. 5, table 2). The components of this model are non-weighted and are simply identified dichotomously as 'yes' or 'no'. With this model the incidence of MT increases from $20 \%$ if the patient has one of these variables present upon admission to $80 \%$ if all four are present. Variables are assigned values of either 0 or 1 based upon whether the value is classified as predictive or not. This algorithm yielded an AUC of 0.839. In an independent internal dataset $(\mathrm{n}=396)$ the ability to accurately identify patients with combat-related injuries in need for MT was 66\% (AUC $=0.747)$. The final predictive equation is: 


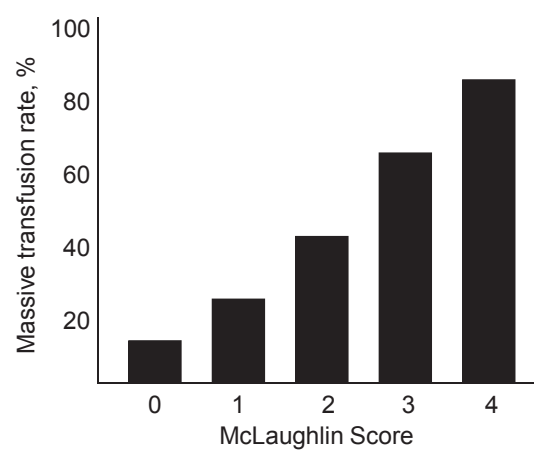

Fig. 5. McLaughlin Score: Observed percentage of MT for each number of variables associated with MT.

\begin{tabular}{|ll|}
\hline ER heart rate $>105 \mathrm{bpm}$ & 1 \\
ER Systolic blood pressure $<110 \mathrm{~mm} \mathrm{Hg}$ & 1 \\
$\mathrm{pH}<7.25$ & 1 \\
Hematocrit $<32 \%$ & 1 \\
\hline
\end{tabular}

$$
\begin{aligned}
& \log (\mathrm{p} /[1-\mathrm{p}])=1,576+(0.825 \times \mathrm{SBP}) \\
& +(0.826 \times \mathrm{HR})+(1.044 \times \mathrm{Hct})+(0.462 \times \mathrm{pH})
\end{aligned}
$$

\section{Larson Model [44]}

This model was developed based upon a retrospective review of 1,124 combat casualties entered between 2003 and 2008 into the Joint Theatre Trauma Registry (JJTR) transfusion database (US Department of Defense database) who had received at least one unit of blood (pRBCs and/or fresh whole blood (FWB), predefined as equivalent) during initial resuscitation [44]. Of these patients, 420 (37\%) had received a MT which was defined as $\geq 10$ units of pRBCs within $24 \mathrm{~h}$ after admission. Four data points were included into the analysis of the patient's likelihood of needing an MT. Based upon a review of the literature, variables found to be predictive for an MT were heart rate $>105 \mathrm{bpm}$, systolic blood pressure $<110$ $\mathrm{mm} \mathrm{Hg}$, hemoglobin $\leq 11 \mathrm{~g} / \mathrm{dl}$, and $\mathrm{BD} \leq 6 \mathrm{mmol} / 1$ [28-30, 34, 45] (fig. 6, table 2). For their model, Larson and colleagues [44] used a cut-off point for heart rate $>110 \mathrm{bpm}$ for ease of remembrance and use. In an attempt to further increase the ease of use for the treating physician, Larson and colleagues [44] also transformed the aforementioned McLaughlin equation into a 'clinical formula'. Both formulas were applied to all patients, and the predicted outcomes were compared with the incidence of MT. The presence of at least two variables produced the most sensitive and specific test (sensitivity 69\%, specificity 65, PPV 54\%, and NPV 78\%) with an incidence for MT of $54 \%$. Patients predicted but not observed to have had MT had an earlier time to death and an increased incidence of head injury compared to those predicted and observed to have had MT. Vice-versa, patients not predicted but observed to have had MT had increased chest, abdominal, and extremity injuries than those neither predicted nor observed to have had an MT.

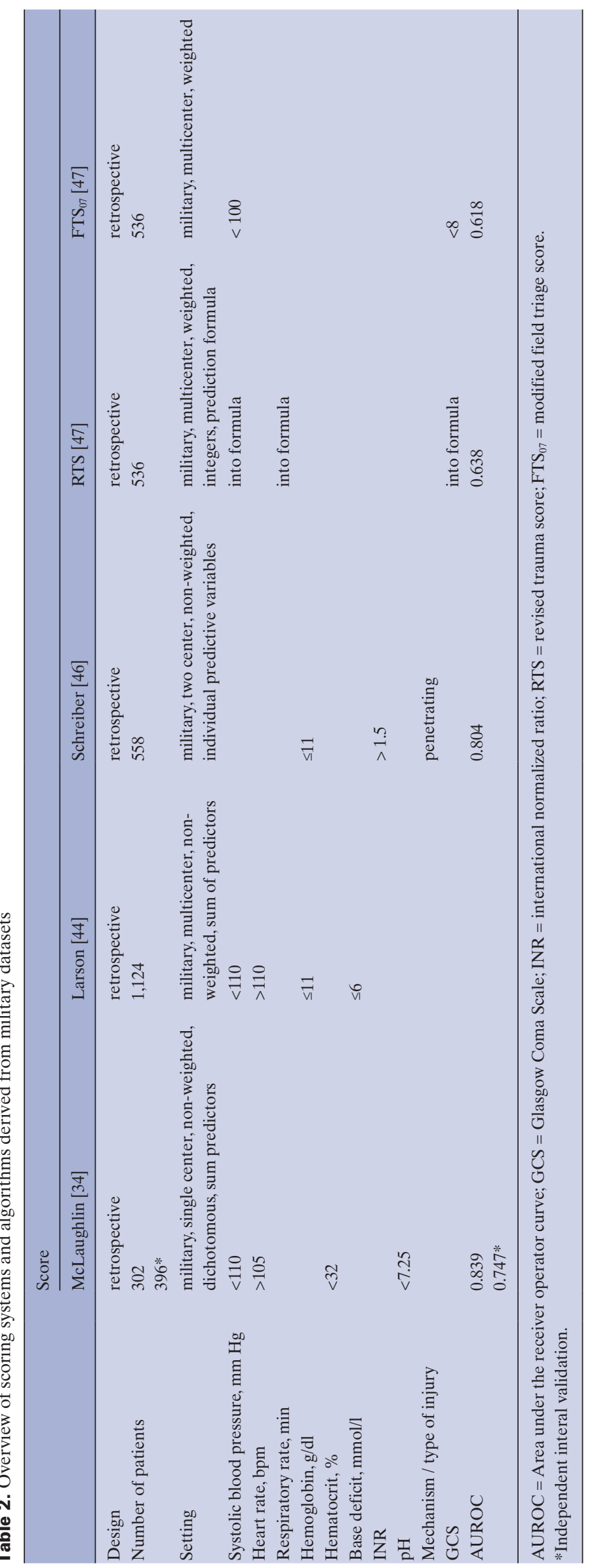

Maegele/Brockamp/Nienaber/Probst/Schoechl/ Görlinger/Spinella 


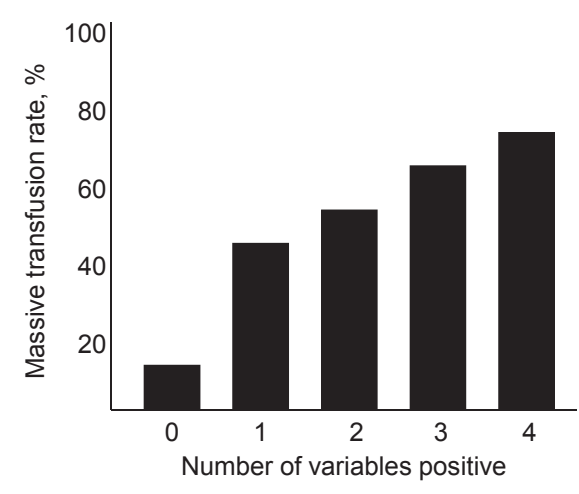

Fig. 6. Larson Model: MT rates associated with multiple variables present in the model.

ER heart rate $>110 \mathrm{bpm}$
ER systolic blood pressure $<110 \mathrm{~mm} \mathrm{Hg}$
Base deficit $(\mathrm{BD}) \leq-6$
Hemoglobin $<11 \mathrm{~g} / \mathrm{gl}$

selected as cut-off based upon the systolic blood pressure at which radial pulse typically switches from strong to weak. In the calculation of $\mathrm{GCS}_{\text {total }}$ no distinction was made between patients who were intubated and those who were not. Likewise, the RR used for score calculation with difference between artificially ventilated and spontaneously breathing patients. Variables different by univariate analysis underwent logistic regression and AUROCs were calculated. By logistic regression analysis, probability of an outcome was given by $\mathrm{p}=$ $\mathrm{e}^{k} /\left(1+\mathrm{e}^{k}\right)$. In result, the predictive accuracy of the equations and of either score was relatively low, with AUROCs of 0.638 (RTS) and $0.618\left(\mathrm{FTS}_{07}\right)$ for prediction of need for MT. The advantage of $\mathrm{FTS}_{07}$ over RTS is its ease of computation.

\section{Discussion}

Exsanguinating hemorrhage is still among the leading causes of death in trauma patients, and the majority of these deaths typically occur within the first $6-12 \mathrm{~h}$ after initial injury [3, 15-18]. Furthermore, 1 out of 4 trauma patients to arrive in the emergency department is already coagulopathic upon admission [810,13 but incidence rates up to $60 \%$ have been reported according to definition [21]. This has led to a new appreciation of the acute coagulopathy in trauma care. Recently, improvements in mortality have been observed in patients who had been ultimately resuscitated with more balanced ratios between pRBCs and fresh frozen plasma (FFP) [24, 25]. In consequence, many trauma centers have altered their MT protocols to reflect this new resuscitation strategy [50], also referred to as 'Damage Control Resuscitation' [51, 52]. Despite substantial improvements in the knowledge on how to adequately resuscitate the exsanguinating patient, one of the remaining and fundamental issues to improve the outcome remains the early identification of patients in the need for transfusion including those requiring MT. Although criteria that trigger the activation of MT protocols remain highly center- and provider-dependent, the benefits of timely MT protocol activation, the identification of appropriate patients given, have been frequently demonstrated $[28,53]$. Early, reliable prediction of the need for MT is necessary to maximize mortality benefits from resuscitation. Vice-versa, there still remains considerable concern about the inappropriate use of MT protocols in patients not in the need of MT as unnecessary exposure of the injured host to blood component therapy may cause harm to this population. Though blood transfusion has the obvious benefit of volume restoration and improved oxygen carrying capacity in the injured patient, there are quite a few risks and immunosuppressive and infectious consequences associated with blood products including transfusion reaction, transmission of bloodborne pathogens, and the impact of limited supply. For these reasons, there has been a trend to restrict transfusion in nonurgent clinical settings and to limit transfusion to ongoing and imminently life-threatening situations. 
Over the past few years, a considerable number of scoring systems have been developed and introduced for the initial evaluation of the bleeding trauma patient in both civilian [28$32,38-43]$ and military settings [34, 44, 46, 47]. The purpose of the present work was to review published data on early predictors as well as scoring systems and algorithms for the need for transfusion including MT after trauma. These systems may provide clinically useful information that potentially gives freedom to providers to deviate from established algorithms toward the more aggressive and early use of blood products with the assumption that early product use improves outcomes. These scoring systems could be used to guide the activation of MT protocols and could help providers of all experience levels know when it is likely that the patient will require a MT. Lastly, these systems may be used in research in stratifying patients for trials of blood substitutes or other interventions and in quantifying the success of such trials.

The scoring systems developed so far usually suggest combinations of physiologic, hemodynamic, laboratory, injury severity, and demographic triggers identified on the initial evaluation of the bleeding trauma patient. Many of the scoring systems presented here use a combination of dichotomous variables that are obtained rapidly in the trauma bay and are readily accessible after the patient's arrival [28-32, 40-43], but others rely on time-consuming mathematical calculations or complex scoring algorithms that are required to determine the patients who will need MT [38, 39] and may thus have limited real-time application.

The most commonly proposed triggers that were correlated with the need for transfusion including MT in the civilian setting are shown in table 1 and include systolic blood pressure which is present in $9 / 9$ scoring systems, followed by heart rate (present in 6/9 scoring systems), hemoglobin/hematocrit (present in 4/9 scoring systems), and FAST positive (present in 4/9 scoring systems). Parameters that can be quickly obtained via point-of-care (POC) arterial blood gas analyzers, for example $\mathrm{BE} / \mathrm{BD}$, lactate and $\mathrm{pH}$, are included in $6 / 9$ civilian scoring systems. Six out of 9 systems consider anatomical injury including its magnitude or mechanism of injury as component of their assessment. However, the severity of injury as reflected by the ISS or the overall pattern of the anatomical injury may be difficult to calculate and to assess during initial assessment.

Interestingly, the INR which reflects the coagulation status as variable to predict transfusion is only present in 2/9 systems. In the majority of systems, rapid availability of the information was a substantial prerequisite for a particular parameter to be potentially considered as a score component. For example, Rainer et al. [31] for their PWH Score intentionally did not consider specific laboratory tests indicating potential presence of coagulopathy, for example hematocrit, prothrombin time (PT), activated partial thromboplastin time (aPTT) and platelet counts, due to unavailability of POC tests in their setting. Vice-versa, with rapid POC testing together with physical ex- amination and easy accessible hemodynamic monitoring the majority of variables for the scores are available during initial trauma work-up and may assist in rapid identification of those at risk for transfusion including MT, thus allowing resources to be quickly activated. In this context, however, it needs to be recognized that INR and BD are good predictors of mortality, but, by themselves, they cannot discriminate those who will (or will not) go on to receive MT. Furthermore, FAST examination depends on the skill of the user and may lose sensitivity due to the body habitus or injury pattern.

The overall accuracy between the scores ranged between AUC 0.804 and AUC 0.905, with weighted scores (TASH and $\mathrm{PWH}$ ) in general performing superior over non-weighted scores. For example, the rates for correct classification for MT were $>90 \%$ with TASH $>16[29,30]$, and $96.9 \%$ with $\mathrm{PWH} \geq$ 6 [31]. For the weighted ETS the probability for transfusion increased exponentially with the sum of ETS points (1 point = $0.7 \%, 3$ points $=5 \%$, maximum 9.5 points $97 \%$ ) $[41,42]$. These authors also defined a cut-off for low risk (probability/ risk of transfusion $\leq 5 \%$ ) at ETS $\leq 3$ with a sensitivity of $97.5 \%$, a specificity of $68 \%$, a PPV of 0.222 , and a NPV of 0.998. For non-weighted scores, the likelihood for MT relied upon the number of predictive triggers present. For example, two or more factors had to be present with the ABC Score before the score would accurately ( $85 \%$ of the time) predict who had received MT [28]. These observations correspond to the recent work by Callcut and colleagues [43], suggesting individual triggers to have differential predictive values for the need of transfusion. These authors had included systolic blood pressure $<90 \mathrm{~mm} \mathrm{Hg}$, hemoglobin $<11 \mathrm{~g} / \mathrm{dl}$, temperature < $35.5^{\circ} \mathrm{C}$, INR $>1.5$, and $\mathrm{BD} \geq 6$ into their analysis and found that the triggers did not contribute equal predictive values for the need of transfusion, with INR being the most predictive for any transfusion and highly predictive for the need of MT. If patients met either INR or systolic blood pressure cut-offs alone, they were likely to receive MT.

For practical combat reasons, the scoring systems and algorithms derived from the military [34, 44, 46, 47] were intentionally kept simple, were in general non-weighted, and included a maximum of four variables as shown in table 2. Similar to the civilian setting, systolic blood pressure was included in $4 / 5$ systems, hemoglobin/hematocrit in $3 / 5$, and heart rate in $2 / 5$. In result, the predictive accuracy of these scores was relatively low with AUROCs ranging between 0.618 and 0.747 .

A major and common limitation to all scoring systems and algorithms identified here, with one exception, is their retrospective nature. All systems have been developed retrospectively based upon datasets derived from single or multicenter civilian or military databases. Some models have been developed using a classical data split approach with half of the dataset used for development and the other half for internal validation [29, 32]. Meanwhile, some scores and algorithms have been internally re-validated on data from the same database, for example the TASH [30] or the McLaughlin Score 


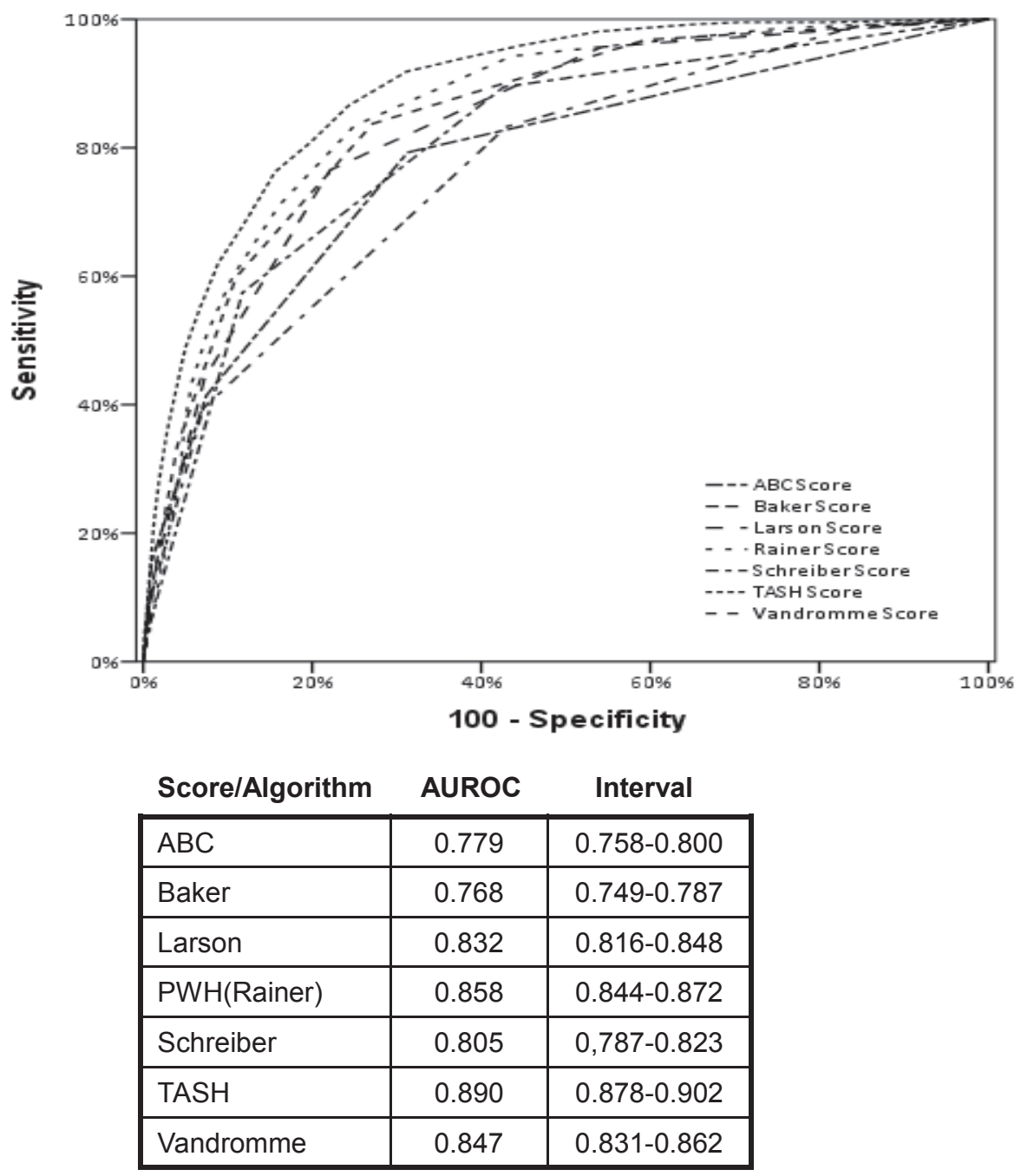

Fig. 7. Validation of seven predictive models on one dataset $(\mathrm{n}=7,042)$ derived from the TR-DGU (Trauma Registry of the Deutsche Gesellschaft für Unfallchirurgie): The TASH Score was internally re-validated while all other scores were externally validated. The two weighted scores (TASH and PWH/Rainer) performed best while ABC Score and Baker Model perfomed less accurate.

[34]. The only score that has been prospectively validated on data from a subset of 481 emergency room patients is the ETS [42]. The Prospective, Observational, Multicenter Massive Transfusion Study (www.uth.tmc.edu/cetir/PROMMTT/) is currently underway and is designed to compare the ability of the Larson Model [44] to predict the need for MT with the predictive ability of the trauma surgeon's clinical judgment.

To date, several systems and algorithms have been applied onto other external but also retrospective datasets and have thus been externally validated. In developing their ABC Score, Nunez et al. [28], for example, have applied both the TASH and the McLaughlin Score onto their local trauma center database including 596 trauma patients for score comparison [28]. In result, all three scores (TASH AUROC = 0.842; McLaughlin AUROC $=0.846$; ABC AUROC $=0.842$ ) were considered as equally good predictors for MT without a statistically significant difference between the scores. In another retrospective study, Cotton and colleagues [54] applied the $\mathrm{ABC}$ Score on adult trauma datasets from three different level I trauma centers in the USA $(\mathrm{n}=513$ from trauma center $1 ; \mathrm{n}=373$ from trauma center 2 ; and $\mathrm{n}=133$ from trauma center 3 ) and compared the predictive ability of the score at each institution. The sensitivity and specificity for the ABC score predicting MT ranged from 75 to $90 \%$ and 67 to $88 \%$, respectively. Correctly classified patients and AUROCs, however, were $84-87 \%$ and $0.83-0.90$, respectively. Recently, Mitra and colleagues [55] compared the performance of the PWH Score [31] to the ABC [28] and TASH Scores [29, 30] by a retrospective review of a subgroup of major trauma patients $(\mathrm{n}=1,234)$ derived from The Alfred Trauma Registry (Victoria/Australia). In this analysis, the performance of the TASH was best with an AUROC of 0.8986 , followed by the PWH Score $(A U R O C=0.8419)$ and the ABC Score.

Our own group recently applied a total of seven scores and algorithms to predict transfusion in trauma patients, i.e. ABC, Baker, Larson, PWH, Schreiber, TASH and Vandromme Scores, onto a large subset of trauma patients derived from the most updated database of the TR-DGU ( $n=7,042$; manuscript in preparation). This extract included data from adult severely injured trauma patients (ISS $>16$ ) with all variables present from each patient to calculate all seven scores. Although we had initially attempted to validate all scores on our database, the remaining scores had to be excluded from this analysis due to missing or non-captured data within our regis- 
try for model calculation. For the TASH Score, this analysis served again as an internal validation while all other scores were externally validated by being subjected to our datasets. Not surprisingly, the TASH performed best (AUROC 0.890) followed by the PWH Score (Rainer Score) which is also a weighted score with structure and content variables very similar to the TASH Score (fig. 7). In this analysis, the nonweighted ABC Score and the Baker Model performed less accurate with AUROCs of 0.779 and 0.768 , respectively.

An alternative to scoring systems and algorithms to early recognize trauma-induced coagulopathy with the risk of ongoing hemorrhage and transfusion requirement is the early use of viscoelastic testing methods [56]. Cotton and colleagues [56] recently presented results from a pilot study in which they had prospectively evaluated the timeliness of real-time rapid thrombelastography results ( $\mathrm{r}-\mathrm{TEG}$ ), their correlation with conventional coagulation tests, and the ability of r-TEG to predict early blood transfusion in 272 consecutive major trauma activations over a 5-month period. Early r-TEG values (activated clotting time (ACT), k-time, and r-value) were available within $5 \mathrm{~min}$ and late $\mathrm{r}$-TEG values (maximal amplitude and $\alpha$-angle) within $15 \mathrm{~min}$, in contrast to results from conventional coagulation testings with turn-around times of 48 min on average. ACT, r-value, and k-time showed strong correlations with later incoming results from conventional testings, and linear regression demonstrated ACT to predict the need for RBCs, plasma, and platelet transfusions within the first $2 \mathrm{~h}$ of arrival. In addition, ACT $<105$ s predicted patients who did not receive any transfusions in the first $24 \mathrm{~h}$. Similar results have been reported by Davenport and colleagues [57]. In their study a threshold of clot amplitude of $\leq 35 \mathrm{~mm}$ at 5 min of rotational thrombelastometry was indicative for acute traumatic coagulopathy and the need for transfusion including MT. This true POC viscoelastic testing may offer the unique potential to predict transfusion even faster as compared to scoring systems involving conventional coagulation testing and to activate and guide resuscitations more objectively. A recent retrospective analysis of major trauma patients revealed low FibTEM amplitudes $(<4 \mathrm{~mm})$ and/or low ExTEM amplitude at $10 \mathrm{~min}$ (CA 10) to be highly predictive for MT [59].

\section{Conclusion}

The early recognition of the acute coagulopathy of trauma accompanied by adequate and aggressive management including the balanced use of blood components can correct coagulopathy, control bleeding, reduce blood product use, and improve outcome in severely injured patients. Despite improvements in knowing how to resuscitate exsanguinating patients, one of the remaining keys is to expeditiously and reproducibly identify the patients most likely to require transfusion including MT. The present work summarizes the yet developed and published algorithms and scoring systems for transfusion including MT in both civilian and trauma populations. The models developed so far usually suggest combinations of physiologic, hemodynamic, laboratory, injury severity, and demographic triggers identified on the initial evaluation of the bleeding trauma patient. Many of the scoring systems use a combination of dichotomous variables that are obtained rapidly in the trauma bay and are readily accessible after the patient's arrival, but others rely on time-consuming mathematical calculations or complex scoring algorithms and may thus have limited realtime application. In general, weighted and more sophisticated systems including higher numbers of variables perform superior over simple non-weighted models. A major and common limitation to all models is their retrospective nature, and prospective validations are urgently needed. In this context the predictive nature of individual triggers should be considered in order to improve the accuracy of the models. The best performance of any model developed to date is reflected by an AUROC of 0.905 and 0.91 (TASH and Vandromme Score). POC viscoelastic testing may be an alternative to these systems to early recognize trauma-induced coagulopathy with the risk of ongoing hemorrhage and transfusion.

\section{Disclosure Statement}

There are no conflicts of interest associated with this article.

\section{References}

1 Krug EG, Sharma GK, Lozano R: The global burden of injuries. Am J Public Health 2000;90:523-526.

2 Murray CJ, Lopez AD: Mortality by cause for eight regions of the world: global burden of disease study. Lancet 1997;349:1269-1276.

$>$ Sauaia A, Moore FA, Moore EE, Moser KS, Brennan R, Read RA, Pons PT: Epidemiology of trauma deaths: a reassessment. J Trauma 1995;38:185-193.

4 Hess JR, Brohi K, Dutton RP, Hauser CJ, Holcomb JB, Kluger Y, Mackway-Jones K, Parr MJ, Rizoli SB, Yukioka T, Hoyt DB, Bouillon B: The coagulopathy of trauma: a review of mechanisms. J Trauma 2008;65:748-754.
5 Brohi K, Cohen MJ, Ganter MT, Matthay MA, Mackersie RC, Pittet JF: Acute traumatic coagulopathy: initiated by hypoperfusion. Ann Surg 2007;245:812-818.

6 Brohi K, Cohen MJ, Davenport R: Acute coagulopathy of trauma: mechanisms, identification and effect. Curr Opin Crit Care 2007;13:680-685.

7 Tieu BH, Holcomb JB, Schreiber MA: Coagulopathy: its pathophysiology and treatment in the injured patient. World J Surg 2007;31:1055-1064.

8 Maegele M: Frequency, risk stratification and therapeutic management of acute post-traumatic coagulopathy. Vox Sang 2009;97:39-49.
9 Brohi K, Singh J, Heron M, Coats T: Acute traumatic coagulopathy. J Trauma 2003;54:1127-1130.

10 Maegele M, Lefering R, Yucel N, Tjardes T, Rixen D, Paffrath T, Simanski C, Neugebauer E, Bouillon B; the AG Polytrauma of the German Trauma Society (DGU): Early coagulopathy in multiply injury: an analysis from the German Trauma Registry on 8724 patients. Injury 2007;38:298-304.

11 Martinowitz U, Michaelson M: Guidelines for the use of recombinant activated factor VII (rFVIIa) in uncontrolled bleeding: a report by the Israeli Multidisciplinary rVIIa Task Force. J Thromb Haemost 2005;3:640-648. 
12 American College of Surgeons: Abdominal trauma.; in Advanced Trauma Life Support ATLS Student Manual, 7th ed. Chicago, American College of Surgeons, 2004, p 137.

13 MacLeod JB, Lynn M, McKenney MG, Cohn SM, Murtha M: Early coagulopathy predicts mortality in trauma. J Trauma 2003;55:39-44.

14 Reed RL, Johnson TD, Hudson JD, Fischer RP: The disparity between hypothermic coagulopathy and clotting studies. J Trauma 1992;33:465-470.

15 Niles SE, McLauglin DF, Perkins JG, et al: Increased mortality associated with the early coagulopathy of trauma in combat casualties. J Trauma 2008;64:1459-1463.

16 Peng R, Chang C, Gilmore D, Bongard F: Epidemiology of immediate and early trauma death at an urban level I trauma center. Am Surg 1998;64:950-954.

17 MacLeod JB, Cohn SM, Johnson EW, McKenney MG: Trauma deaths in the first hour: are they all unsalvageable injuries? Am J Surg 2007;193:195-199.

18 Demitriades D, Murray J, Charalambides K, et al: Trauma fatalities: time and location of hospital deaths. J Am Coll Surg 2004;198:20-26.

19 Como JJ, Dutton RP, Scalea TM, Edelman BB, Hess JR: Blood transfusion rates in the care of acute trauma. Transfusion 2004;44:809-813.

20 Malone DL, Dunne J, Tracy JK, Putman AT, Scalea TM, Napoletano LM: Blood transfusion, independent of shock severity, is associated with worse outcome in trauma. J Trauma 2003;54:898-905.

21 Floccarda B, Rugerib L, Faurea A, et al: Early coagulopathy in trauma patients: an on-scene and hospital admission study. Injury 2012;43:26-32.

-22 Repine TB, Perkins JG, Kauvar DS, Blackborne L: The use of fresh whole blood in massive transfusion. J Trauma 2006;60:S59-S69.

-23 Kauvar DS, Holcomb JB, Norris GC, Hess JR: Fresh whole blood transfusion: a controversial military practice. J Trauma 2006;61(6 suppl):181-184.

-24 Borgman M, Spinella PC, Perkins JG, Grathwoh KW, Repine T, Beekley AC, Sebesta J, Jenkins D Wade CE, Holcomb JB: The ratio of blood products transfused affects mortality in patients receiving massive transfusions at a combat support hospital. J Trauma 2007;63:805-813.

25 Maegele M, Lefering R, Paffrath T, Tjardes T, Simanski C, Bouillon B; the Working Group on Polytrauma of the German Society of Trauma Surgery (DGU): Red blood cell to plasma ratios transfused during massive transfusion are associated with mortality in severe multiply injury. A retrospective analysis from the Trauma Registry of the Deutsche Gesellschaft für Unfallchirurgie. Vox Sang 2008;95:112-119.

-26 Karkouti K, O'Farrell R, Yau TM, Beattie WS: Predication of massive blood transfusion in cardiac surgery. Can J Anesth 2006;53:781-794.

27 McCluskey SA, Karkouti K, Wijeysundra DN, et al: Derivation of a risk index for the prediction of massive blood transfusion in liver transplantation. Liver Transpl 2006;12:1584-1593.

28 Nunez TC, Voskresensky IV, Dossett LA, Shinall R, Dutton WD, Cotton BA: Early prediction of massive transfusion in trauma: simple as $\mathrm{ABC}$ (Assessment of Blood Consumption)? J Trauma 2009;2:346-352.

-29 Yucel N, Lefering R, Maegele M, Vorweg M, Tjardes T, Ruchholtz S, Neugebauer E, Wappler F, Bouillon B, Rixen D; the Polytrauma Study Group of the German Trauma Society: Trauma Associated Severe Haemorrhage (TASH)-Score: probability of mass transfusion as surrogate for life threatening haemorrhage after multiple trauma. J Trauma 2006;60:1228-1237.
30 Maegele M, Lefering R, Wafaisade A, Theodorou P, Wutzler S, Fischer P, Bouillon B, Paffrath T; the Trauma Registry of the Deutsche Gesellschaft für Unfallchirurgie (TR-DGU): Revalidation and update of the TASH-Score: a scoring system to predict the probability for massive transfusion as a surrogate for life-threatening haemorrhage after severe injury. Vox Sang 2011;100:231-238.

31 Rainer TH, Ho AMH, Yeung JHH, Cheung NK, Wong RSM, Tang N, Ng SK, Wong GKC, Lai PBS, Graham CA: Early risk stratification of patients with major trauma requiring massive blood transfusion. Resuscitation 2011;82:724-729.

32 Vandromme MJ, Griffin RL, McGwin G Jr, Weinberg JA, Rue LW 3rd, Kerby JD: Prospective identification of patients at risk for massive transfusion. Am Surg 2011;77:155-161.

33 Schreiber MA, Perkins J, Kiraly L, Underwood S, Wade C, Holcomb JB: Early predictors of massive transfusion in combat casualties. J Am Coll Surg 2007;205:541-545.

34 McLaughlin DF, Niles S, Salinas J, Perkins JG, Cox ED, Wade C, Holcomb JB: A predictive model for massive transfusion in combat casualty patients. $\mathrm{J}$ Trauma 2008;64(2 suppl):S57-S63.

35 Cancio LC, Wade C, West SA, Holcomb JB: Prediction of mortality and of the need for massive transfusion in casualties arriving at combat support hospitals in Iraq. J Trauma 2008;64(2 suppl):S51S55.

36 Vandromme MJ, Griffin RL, Weinberg JA, Rue LW 3rd, Kerby JD : Lactate is a better predictor than systolic blood pressure for determining blood requirement and mortality: could prehospital measures improve trauma triage? J Am Coll Surg 2010;210:861-869.

37 Rothmann KJ: Epidemiology: An Introduction. New York, Oxford University Press, 2002.

38 Wade CE, Holcomb JB, Chrisholm GB, Michalek JE: Accurate and early prediction of massive transfusion in trauma patients. 67th Annual Meeting of the American Association for the Surgery in Trauma, Maui (Hawaii), 2008, abstract.

39 Moore F, McKinley B, Moore E, Nathens A, Rhee P, Puyana J, Beilman G, Cohn S: Need for massive transfusion can be predicted early after trauma center arrival. J Trauma 2007;62:270(abstract).

40 Baker JB, Korn CS, Robinson K, Chan L, Henderson SO: Type and crossmatch of the trauma patient. J Trauma 2001;50:878-881.

41 Ruchholtz S, Pehle B, Lewan U, Lefering R, Müller $\mathrm{N}$, Oberbeck R, Waydhas C: The emergency room transfusion score (ETS): prediction of blood transfusion requirement in initial resuscitation after sever trauma. Transfusion Med 2006;16:49-56.

42 Kühne C, Zettl RP, Fischbacher M, Lefering R, Ruchholtz S: Emergency Transfusion Score (ETS): a useful instrument for prediction of blood transfusion requirement in severely injured patients. World J Surg 2006;32:1183-1188.

43 Callcut RA, Johannigman JA, Kadon KS, Hanseman DJ, Robinson BRH: All massive transfusion criteria are not created equal: defining the predictive value of individual transfusion triggers to better determine who benefits from blood. J Trauma 2011;70:794-801.

44 Larson CR, White CE, Spinella PC, Jones JA, Holcomb JB, Blackbourne LH, Wade CE: Association of shock, coagulopathy, and initial vital signs with massive transfusion in combat casualties. J Trauma 2010;69(suppl 1):S26-32.
45 Eastridge BJ, Salinas J, McManus JG, et al: Hypotensions begins at $110 \mathrm{mmHg}$ : redefining 'hypotension' with data. J Trauma 2007;63:291-297.

46 Schreiber MA, Perkins J, Kiraly L, Underwood S, Wade C, Holcomb JB: Early predictors of massive transfusion in combat casualties. J Am Coll Surg 2007;205:541-545.

47 Cancio LC, Wade CE, West SA, Holcomb JB: Prediction of mortality and of the need for massive transfusion in casualties arriving at combat support hospitals in Iraq. J Trauma 2008;64(2 suppl):S51S56.

48 Champion HR, Sacco WJ, Copes WS, Gann DS, Gennarelli TA, Flanagan ME: A revision of the Trauma Score. J Trauma 1989;29:623-629.

49 Eastridge BJ, Butler F, Wade CE, Holcomb JB, Salinas J, Champion HR, Blackbourne LH: Field triage score (FTS) in battlefield casualties: validation of a novel triage technique in a combat environment. Am J Surg 2010;200:724-727.

50 Gonzales EA, Moore FA, Holcomb JB, Miller CC, Kozar RA, Todd SR, Cocanour CS, Balldin BC, McKinley BA: Fresh frozen plasma should be given earlier to patients requiring massive transfusion. J Trauma 2007;62:112-119.

51 Holcomb JB, Jenkins D, Rhee P, Johannigman J, Mahoney P, Mehta S, Cox ED, Gehrke MJ, Beilman GJ, Schreiber M, Flaherty SF, Grathwohl KW, Spinella PC, Perkins JG, Beekley AC, McMullin NR, Park MS, Gonzales EA, Wade CE, Dubick MA, Schwab CW, Moore FA, Champion HR, Hoyt D, Hess J: Damage control resuscitation: directly addressing the early coagulopathy of trauma. J Trauma 2007;62:307-310.

52 Holcomb JB: Damage control resuscitation. J Trauma 2007;62(6 suppl) :S36-S37.

53 Zink KA, Sambasivan CN, Holcomb JB, Chrisholm G, Schreiber MA: A high ratio of plasma and platelets to packed red blood cells in the first 6 hours of massive transfusion improves outcome in a large multicenter study. Am J Surg 2009;197:565-570.

54 Cotton BA, Dossett LA, Haut ER, Shafi S, Nunez TC, Au BK, Zaydfudim V, Johnston M, Arbogast P, Young PP: Multicenter validation of a simplified score to predict massive transfusion in trauma. $\mathrm{J}$ Trauma 2010;69(suppl 1):S33-39.

55 Mitra B, Rainer T, Cameron P: Predicting massive blood transfusion post trauma. Resuscitation 2011; doi: 10.1111/j.1423-0410.2011.01564.x.

56 Cotton BA, Faz G, Hatch QM, Radwan ZA, Podbielski J, Wade C, Kozar RA, Holcomb JB: Rapid thrombelastography delivers real-time results that predict transfusion within 1 hour of admission. J Trauma 2011;71:407-417.

57 Davenport R, Manson J, De'Ath H, Platton S, Coates A, Allard S, Hart D, Pearse R, Pasi KJ, Maccallum P, Stanworth S, Brohi K: Functional definition and characterization of acute traumatic coagulopathy. Crit Care Med 2011;39:2652-2658.

58 Meißner A, Schlenke P: Massive bleeding and transfusion. Transfus Med Hemother 2012;39(2): 73-84.

59 Schoechl H, Cotton B, Inaba K, Nienaber U, Fischer H, Voelkl W, Solomon C: FibTEM provides early prediction of massive transfusion in trauma. Crit Care 2011;15:R265. 